\title{
Fluxon interaction with external rf radiation in Josephson junctions
}

\author{
Kivshar, Yuri S.; Olsen, Ole H.; Samuelsen, Mogens Rugholm; Ustinov, Alexey V.
}

Published in:

Physical Review B

Link to article, DOI:

10.1103/PhysRevB.47.5212

Publication date:

1993

Document Version

Publisher's PDF, also known as Version of record

Link back to DTU Orbit

Citation (APA):

Kivshar, Y. S., Olsen, O. H., Samuelsen, M. R., \& Ustinov, A. V. (1993). Fluxon interaction with external rf radiation in Josephson junctions. Physical Review B, 47(9), 5212-5218.

https://doi.org/10.1103/PhysRevB.47.5212

\section{General rights}

Copyright and moral rights for the publications made accessible in the public portal are retained by the authors and/or other copyright owners and it is a condition of accessing publications that users recognise and abide by the legal requirements associated with these rights.

- Users may download and print one copy of any publication from the public portal for the purpose of private study or research.

- You may not further distribute the material or use it for any profit-making activity or commercial gain

- You may freely distribute the URL identifying the publication in the public portal

If you believe that this document breaches copyright please contact us providing details, and we will remove access to the work immediately and investigate your claim 


\title{
Fluxon interaction with external rf radiation in Josephson junctions
}

\author{
Yuri S. Kivshar* \\ Institut für Theoretische Physik I, Heinrich-Heine-Universität Düsseldorf, D-4000 Düsseldorf 1, Germany \\ Ole H. Olsen \\ Biostructure Department, Novo-Nordisk Research Institute, DK-2880 Bagsværd, Denmark \\ Mogens R. Samuelsen and Alexey V. Ustinov ${ }^{\dagger}$ \\ Physics Laboratory I, The Technical University of Denmark, DK-2800 Lyngby, Denmark
}

(Received 20 August 1992)

\begin{abstract}
Interaction of a fluxon with an rf radiation emitted into a long Josephson junction is investigated analytically and numerically. We use a model based on the sine-Gordon equation driven by a periodic force at the boundary with the frequency larger than the plasma frequency of the junction. It is shown that due to excitation of a standing linear wave by the driving force, the fluxon motion is strongly influenced by a periodic (averaged) potential similar to the Peierls-Nabarro potential in a discrete chain. This effective potential decreases in the direction of the boundary where the external rf drive is applied, so that the fluxon always moves to the active boundary. We calculate the fluxon parameters and confirm our analytical predictions by direct numerical simulations.
\end{abstract}

\section{INTRODUCTION}

As is well known, the dynamics of long Josephson junctions shows a rich variety of interesting nonlinear phenomena, which have been observed experimentally and explained analytically (see, e.g., Ref. 1 and references therein). In particular, the dynamics of fluxons in quasi-one-dimensional Josephson structures has received increasing interest over the last decade as a model system for soliton physics and also because of possible applications for microwave generation. ${ }^{2}$ When an external rf signal is applied to the junction, it creates a set of interesting features in nonlinear dynamics; e.g., depending on the amplitude of the rf signal, various nonlinear phenomena such as hysteresis, period doubling sequences, quasiperiodicity, etc., have been predicted analytically in the framework of the model based on a perturbed sineGordon (SG) equation. ${ }^{3-6}$ As has been shown, when the frequency of the rf signal is within the spectrum gap of the linear (plasmon) waves, the most interesting effect of the applied signal is the hysteresis loop which consists of an upper and lower branch, respectively. ${ }^{3}$ These two branches may be explained in the framework of a simple approach which assumes that the excited localized nonlinear oscillation at the junction edge may be considered as an effective breather mode, so that the breather dynamics may be studied in the framework of the collectivecoordinate approach as that of an effective nonlinear oscillator showing a nonlinear resonance and hysteresis. ${ }^{7}$

Recently, a series of experimental studies of the fluxon dynamics in long Josephson junctions has been initiated to analyze the influence of external millimeter-wave irradiation on the flux-flow dynamics. ${ }^{8,9}$ In particular, a type of resonant flux-flow satellite steps was observed which can be interpreted as parametric mixing of the flux-flow oscillations with the external rf signal; it was suggested that a natural qualitative explanation for these steps was the microwave-assisted reflections of fluxons into antifluxons at the junction boundary. ${ }^{8}$

In the present paper, mostly motivated by the experimental studies in Refs. 8 and 9, we analyze the effect of the external rf signal on the dynamics of a fluxon $(2 \pi$ fluxon) in the framework of the perturbed SG equation with the external ac force applied as a boundary condition. We assume that the signal is applied at a frequency which is above the spectrum gap; i.e., it generates linear waves (plasmons) in the junction. The linear waves created at the edge of the junction propagate along it and influence the fluxon dynamics. We investigate the motion of the fluxon and find the dependence of the fluxon parameters on the parameters of the external rf signal, combining analytical and numerical approaches. In particular, we demonstrate that due to the external rf signal the slow (time-averaged) motion of the fluxon is influenced by a periodic (averaged) potential similar to the Peierls-Nabarro potential in the dynamics of the discrete SG chain and this potential is decreasing to the boundary where the external rf drive is applied, so that the fluxon always moves towards the active boundary. We confirm these conclusions by direct numerical simulations and observe a rather good agreement between the analytical predictions based on the method of averaging and numerical results.

The paper is organized as follows. Section II contains an outline of the model which is based on the SG equation with the ac driving force applied as a boundary condition. In Sec. III we describe the analytical method developed to analyze the problem. The method itself is rather gen- 
eral to be applied to various nonlinear problems which involve a rapidly oscillating ac drive at a boundary. Section IV is devoted to the analysis of the fluxon motion under the influence of the driving force. We derive a dynamical equation for the fluxon coordinate and we show that the motion of the fluxon may be described as that of a particle in an effective (averaged) potential. In Sec. V results of numerical solution of the model equation are presented and they are compared with our analytical predictions showing a good agreement for large frequencies. Section VI concludes the paper summarizing the main results.

\section{MODEL}

The mathematical model used to describe the dynamics of a long Josephson junction is the perturbed SG equation including a dissipative term,

$$
\phi_{t t}-\phi_{x x}+\sin \phi=-\alpha \phi_{t},
$$

where $\phi$ is the phase difference between the quantum mechanical wave functions of the superconductors defining the junction. The spatial coordinate $x$ is normalized to the Josephson penetration depth $\lambda_{J}=\left(h / 2 e d \mu_{0} J\right)^{1 / 2}$, and time is normalized to the inverse plasma frequency $\omega_{0}^{-1}=(2 e J / h C)^{1 / 2}$. Here $J$ is the Josephson current density, $d$ is the magnetic thickness of the barrier, and $C$ is the capacitance per unit area. The loss parameter $\alpha$ models dissipative (quasiparticle) current, and it is defined through the shunt conductance $G$ per unit area, $\alpha=G(h / 2 e J C)^{1 / 2}$.

When an oscillatory magnetic field $H_{e} \cos (\omega t)$ is applied to one edge (in our case at $x=L$ ) of the junction, perpendicular to the length of the junction and parallel to the plane of the barrier, the boundary conditions for the phase difference $\phi$ at the edges are (see, e.g., Refs. 3-7),

$$
\left.\phi_{x}\right|_{x=0}=0,\left.\quad \phi_{x}\right|_{x=L}=a \cos (\omega t),
$$

where $a=H_{e} / J \lambda_{J}$ and $L=l / \lambda_{J}$ are the normalized magnetic field strength and the junction length, respectively. As in Refs. 3-7, we assume that the influence of an external magnetic field is felt only through the boundary conditions such as in Eq. (2). In experimental studies, ${ }^{8,9}$ the external rf signal has been applied at a frequency which is larger than the plasma frequency $[=1$ in the dimensional variables as it is in Eq. (1)]. This condition is different to that considered in Refs. 3-7, because such a signal generates linear waves (plasmons) which propagate along the junction. The problem we are going to analyze is how the linear waves induced by the external rf signal will influence the fluxon motion in the junction. In particular, in this paper we restrict ourselves to the case where the fluxon is initially at rest, so that we are interested in the effect of the rf radiation on the fluxon parameters and its motion.

The problem formulated above allows us to use a quite general formalism which may be extended to a variety of different problems involving an external high-frequency driving force at the boundary. The approach we apply here is based on the method of averaging of nonlinear dynamics and it may be considered as a version of mean field theory. The similar approach has been recently used in two other problems related to the kink dynamics in the SG and $\phi^{4}$ models perturbed by a parametric external force which was taken to be homogeneous in space. ${ }^{10,11}$

\section{AVERAGED EQUATION OF MOTION}

The ac force applied at the boundary of the junction generates a linear wave which propagates along the junction and, after a reflection from another boundary, a standing linear wave forms which subsequently influences the fluxon dynamics. Because the frequency of the standing wave is large $(\omega>1)$, we will apply an averaging method analogous to the well-known one for the Kapitza problem, i.e., for the dynamics of a pendulum hanging from an oscillating suspension point (see Ref. 12). In order to derive an averaged equation of motion in our case, we will decompose the wave field $\phi$ into a sum of slow and fast varying parts, that is to say,

$$
\phi=\Phi+\psi,
$$

where the functions $\Phi(x, t)$ and $\psi(x, t)$ describe the slow and fast evolutions, respectively. The function $\psi(x, t)$ stands for small oscillations around the slowly varying field $\Phi$, and the mean value of $\psi$ during the period $2 \pi / \omega$ is assumed to be zero, i.e., $\langle\phi\rangle=\Phi$, the brackets standing for time average. Our goal is to derive an effective equation for the function $\Phi$ that describes the slow ("averaged") wave field dynamics. To this end, we substitute Eq. (3) into Eqs. (1) and (2), and expanding in $\psi$, which we assume to be small enough for such a purpose, we obtain the equation

$$
\begin{aligned}
\Phi_{t t}+\psi_{t t}-\Phi_{x x}-\psi_{x x}+\sin \Phi+\psi \cos \Phi & -\frac{1}{2} \psi^{2} \sin \Phi \\
& =-\alpha \Phi_{t}-\alpha \psi_{t}
\end{aligned}
$$

where we neglect higher-order harmonics contributions. The boundary conditions are

$$
\begin{aligned}
& \left.\left(\Phi_{x}+\psi_{x}\right)\right|_{x=0}=0 \\
& \left.\left(\Phi_{x}+\psi_{x}\right)\right|_{x=L}=a \cos \omega t .
\end{aligned}
$$

Let us go on taking a careful look at Eqs. (4)-(6). It is clear that the equations have terms of a very different nature, slow and fast varying ones. Hence, the fast and slow terms must satisfy Eqs. (4)-(6) separately. In order to satisfy the rapidly oscillating parts in Eqs. (4)-(6), it is necessary to take into account all the terms which are proportional to the rapidly varying function $\psi$ plus the term $\sim a \cos (\omega t)$, which is also fast. As a result, the following equations must hold for the rapidly oscillating 
part:

$$
\begin{aligned}
& \psi_{t t}-\psi_{x x}+\omega_{0}^{2} \psi=-\alpha \psi_{t} \\
& \left.\psi_{x}\right|_{x=0}=0,\left.\quad \psi_{x}\right|_{x=L}=a \cos (\omega t)
\end{aligned}
$$

where $\omega_{0}^{2}=\cos \Phi$. Now, as the function $\Phi$ evolves much slower than $\psi$, we can consider the function $\omega_{0}^{2}$ in Eq. (7) as constant, and subsequently write the forced solution of the linear equations ( 7 ) in the form,

$\psi(x, t)=a \cos \omega t\left[A_{1} \cos k_{1} x \cosh k_{2} x+A_{2} \sin k_{1} x \sinh k_{2} x\right]+a \sin \omega t\left[A_{2} \cos k_{1} x \cosh k_{2} x-A_{1} \sin k_{1} x \sinh k_{2} x\right]$,

where

$$
\begin{aligned}
& A_{1}=\frac{\Delta_{1}}{\Delta_{1}^{2}+\Delta_{2}^{2}}, \quad A_{2}=\frac{\Delta_{2}}{\Delta_{1}^{2}+\Delta_{2}^{2}} \\
& \Delta_{1,2}=k_{1,2} \sin k_{1} L \cosh k_{2} L \mp k_{2,1} \cos k_{1} L \sinh k_{2} L
\end{aligned}
$$

and the parameters $k_{1}$ and $k_{2}$ are the real and imaginary parts of the wave number, $k=k_{1}+i k_{2}$, which are defined by the expression $k^{2}=\omega^{2}-\omega_{0}^{2}+i \alpha \omega$, i.e.,

$$
k_{1,2}^{2}=\frac{1}{2}\left[ \pm\left(\omega^{2}-\omega_{0}^{2}\right)+\sqrt{\left(\omega^{2}-\omega_{0}^{2}\right)^{2}+\alpha^{2} \omega^{2}}\right] .
$$

In the limit $\alpha \rightarrow 0$ we have $k_{2} \rightarrow 0$, and the solution (8) transforms to a more simple one

$$
\psi(x, t)=-\frac{a}{k \sin k L} \cos \omega t \cos k x,
$$

where $k^{2}=\omega^{2}-\omega_{0}^{2}$.

The next stage of our analysis is to input Eqs. (8)-(11) into Eq. (4) and to average over the fast oscillations. By doing so we derive the equation for the slowly varying function $\Phi$, which turns out to be

$$
\Phi_{t t}-\Phi_{x x}+\left(1-\frac{1}{2}\left\langle\psi^{2}\right\rangle\right) \sin \Phi=-\alpha \Phi_{t},
$$

with the boundary conditions

$$
\left.\Phi_{x}\right|_{x=0}=\left.\Phi_{x}\right|_{x=L}=0,
$$

where the function $\left\langle\psi^{2}\right\rangle$ has to be calculated with the help of Eq. (8) and finally it looks quite simple,

$$
\left\langle\psi^{2}\right\rangle=\frac{a^{2}\left[\cosh \left(2 k_{2} x\right)+\cos \left(2 k_{1} x\right)\right]}{2\left(k_{1}^{2}+k_{2}^{2}\right)\left[\cosh \left(2 k_{2} L\right)-\cos \left(2 k_{1} L\right)\right]} .
$$

In the limit $\omega^{2} \gg 1$, the wave numbers are also large, in particular, $k_{1}^{2} \gg 1$; i.e., the spatial dependence of $\left\langle\psi^{2}\right\rangle$ will be also rapidly oscillating, but now also in $x$. Thus, after averaging Eq. (15) in $x$, the last (oscillating) term in the right-hand side (RHS) of the equation will disappear, and the dependence $\left\langle\psi^{2}\right\rangle$ vs $x$ is smoothed. In the general case the wave number $k$ is not large, even though the driving frequency is large, and the function $\left\langle\psi^{2}\right\rangle$ is spatially oscillating with the wavelength $\lambda=\pi / k_{2}$. Thus, the renormalized SG equation (13) is the main result of our analytical approach, and it describes an averaged nonlinear dynamics of the junction in the case where the external rf signal excites a standing linear wave.

\section{FLUXON DYNAMICS INDUCED BY RADIATION}

In a nondissipative case, when $\alpha=0$, the resulting Eq. (13) is the SG equation with the spatially varying coefficient,

$$
\Phi_{t t}-\Phi_{x x}+\Omega^{2}(x) \sin \Phi=0,
$$

where $\Omega^{2}(x) \equiv\left(1-\frac{1}{2}\left\langle\psi^{2}\right\rangle\right)$. In the system with an infinite length, Eq. (16) has an exact fluxon solution of the form

$$
\Phi=4 \tan ^{-1} \exp \left(\Omega \frac{x-X_{0}}{\sqrt{1-\dot{X}_{0}^{2}}}\right),
$$

provided $\Omega^{2}(x)=\Omega^{2}=$ const. Here $X_{0}=X_{0}(t)$ is the fluxon position, $\dot{X}_{0}$ is the fluxon velocity, and the fluxon energy is given by the expression

$$
E_{k}=8 \Omega / \sqrt{1-\dot{X}_{0}^{2}}
$$

In the subsequent analysis we assume that the system length $L$ is much larger than the fluxon width $l_{k} \propto \Omega^{-1}$, so that even in the long- but finite-length system we may use the solution (17) for the infinite model, assuming that the fluxon coordinate is not at the system boundary. When the function $\Omega^{2}(x)$ is spatially dependent, but slowly varying in $x$, we may consider the fluxon dynamics adiabatically, assuming that the fluxon shape is given by Eq. (17), but its parameters are slowly varying in $t$. Then, as follows from Eqs. (15) and (16), the inequality $\Omega^{2}(x)>\Omega^{2}(L)$ is valid, so that $E_{k}(x)>E_{k}(L)$. Therefore, the fluxon always has the lower energy at the boundary where the external ac force is applied. Moving along the junction, the fluxon changes its width, which may approximately be described by the relation [see Eq. (17)]

$$
l_{k} \approx \Omega^{-1} \sqrt{1-\dot{X}_{0}^{2}} \sim \Omega^{-1}(x) .
$$

To derive the equation of motion for the fluxon coordinate $X_{0}$, we use the simplest version of the perturbation theory for solitons which is based on the use of conservation laws (see, e.g., Ref. 13 for a review and Ref. 14 for a concrete problem involving conservative perturbations). To apply this approach, we write the SG equation (13) in the form

$$
\Phi_{t t}-\Phi_{x x}+\sin \Phi=A(x) \sin \Phi-\alpha \Phi_{t},
$$

where $A(x)=\frac{1}{2}\left\langle\psi^{2}\right\rangle$ is given by Eq. (15) and it is considered as a perturbation, additionally to the dissipative 
term $(\sim \alpha)$. The simplest way to proceed now is to use conservation laws which are modified by perturbations. Let us consider the momentum of the wave field $\Phi$ defined as

$$
P=-\int_{-\infty}^{\infty} \Phi_{t} \Phi_{x} d x
$$

In the SG system without perturbations [i.e., when the RHS of Eq. (20) is zero], the momentum (21) is conserved, because $P$ is an integral of motion. In the presence of perturbations the equation describing the perturbation-induced evolution of the momentum is given by

$$
\frac{d P}{d t}=-\int_{-\infty}^{\infty} d x A(x) \Phi_{x} \sin \Phi-\alpha P .
$$

Equation (22) is exact; however, it may be effectively used only to develop a perturbative approach. Let us assume that the function $A(x)$ is slowly varying in $x$. This is valid, e.g., for the case of large $k_{2}$, when the function $A(x)$ may be considered as averaged with respect to fast spatial oscillations. The slow dependence of $A(x)$ on $x$ assumes that Eq. (22) may be transformed to be of the form

$$
\frac{d P}{d t}=-4 A^{\prime}\left(X_{0}\right)-\alpha P,
$$

where the prime stands for the derivative. To obtain Eq. (23), we have used, as an approximation, the fluxon shape $u_{k}=4 \tan ^{-1} \exp \left(x-X_{0}\right)$. Calculating the fluxon momentum as $P \approx 8 \dot{X}_{0}$, we finally get the motion equation for the fluxon coordinate $X_{0}$,

$$
\frac{d^{2} X_{0}}{d t^{2}}=-\frac{d U_{\text {eff }}}{d X_{0}}-\alpha \frac{d X_{0}}{d t}
$$

Equation (24) describes the motion of a particle of the mass $m=1$ under the dissipative force $(\sim \alpha)$ and in the effective potential

$$
U_{\text {eff }}\left(X_{0}\right) \approx-\left.\frac{1}{4}\left\langle\psi^{2}\right\rangle\right|_{x=X_{0}},
$$

where $\left\langle\psi^{2}\right\rangle$ is defined as an averaged (in time) spatially dependent function. In a more general case the effective potential may be defined from Eq. (22) as

$$
U_{\mathrm{eff}}\left(X_{0}\right) \approx-\frac{1}{8} \int_{-\infty}^{\infty} d z \frac{A^{\prime}\left(z+X_{0}\right)}{\cosh ^{2} z}
$$

As follows from Eq. (25), the properties of the effective potential $U_{\text {eff }}$ are completely determined by the averaged function $\left\langle\psi^{2}\right\rangle$. Because this function is oscillatory increasing, the effective potential is decreasing, so that the fluxon, even being initially at rest, will move to the active boundary under the action of the driving force applied at the edge of the junction. Figure 1 shows the averaged function $\left\langle\psi^{2}\right\rangle$ for constant value of the amplitude of the applied microwave signal, $a=0.5$, the frequency is varied from $\omega=1$ to $\omega=5$, the length of the junction is $L=20$, and the fluxon is placed in $X_{0}=10$. The function $\left\langle\psi^{2}\right\rangle$ has been calculated using the definition given by Eq. (26)

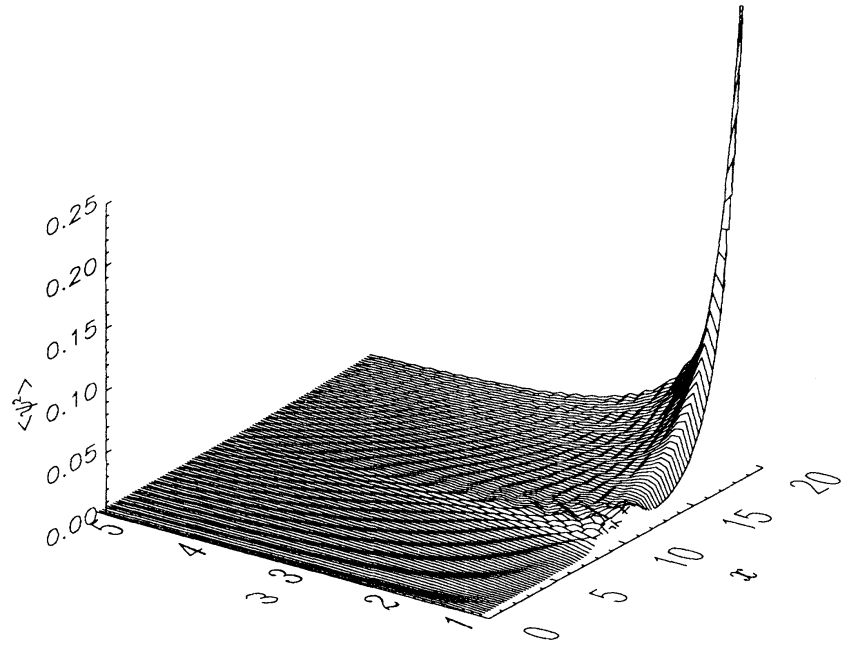

FIG. 1. Averaged field $\left\langle\psi^{2}\right\rangle$ as a function of $x$ and $\omega$ for the length of the junction $L=20$; the $2 \pi$ fluxon is placed at $X_{0}=10$. The microwave source is placed at the right end of the junction (at $x=20$ ). The resulting effective potential given by Eq. (25) has a character which eventually forces the fluxon to move towards the right boundary.

where $\omega_{0}=\cos \Phi, \Phi$ being the usual expression for the fluxon ( $2 \pi \mathrm{kink})$ solution. The increase of $\left\langle\psi^{2}\right\rangle$ for increasing $x$ results in an effective potential (which is just of opposite sign to $\left\langle\psi^{2}\right\rangle$ ) which attracts fluxons placed in the junction.

\section{NUMERICAL SIMULATION RESULTS}

In the present section the results obtained from the perturbation approach are compared to results from the numerical solution of the SG equation describing the interaction between the fluxon and radiation emitted by the microwave source placed at the boundary. Throughout this paper, the loss term $\alpha=0.2$. The microwave source is placed in the right end of the junction.

First, examples of fluxon dynamics confirm the prediction of the existence of an effective attractive potential. Fluxons are shown to be attracted by the active boundary in the full SG system. Second, a comparison between trajectories determined from numerical solution of the full system and the perturbation approach is performed.

In order to illustrate the effect of the predicted attracting potential derived in the former section, two examples of fluxon dynamics are shown in Fig. 2 in terms of contour plots of the spatial derivative $\phi_{x}$. The fluxon is initially placed in $X_{0}(0)=10$. Because of the interaction with the radiation penetrating the junction, the fluxon moves to the right. In Fig. 2(a) the parameter values are $\omega=2$ and $a=0.5$ while in Fig. 2(b) they are $\omega=8$ and $a=0.5$. The interaction between the radiation and the fluxons is clearly seen. For the lower value of the frequency $\omega$, the fluxon has been attracted to the right boundary. The attraction is much less pronounced for the larger value of 

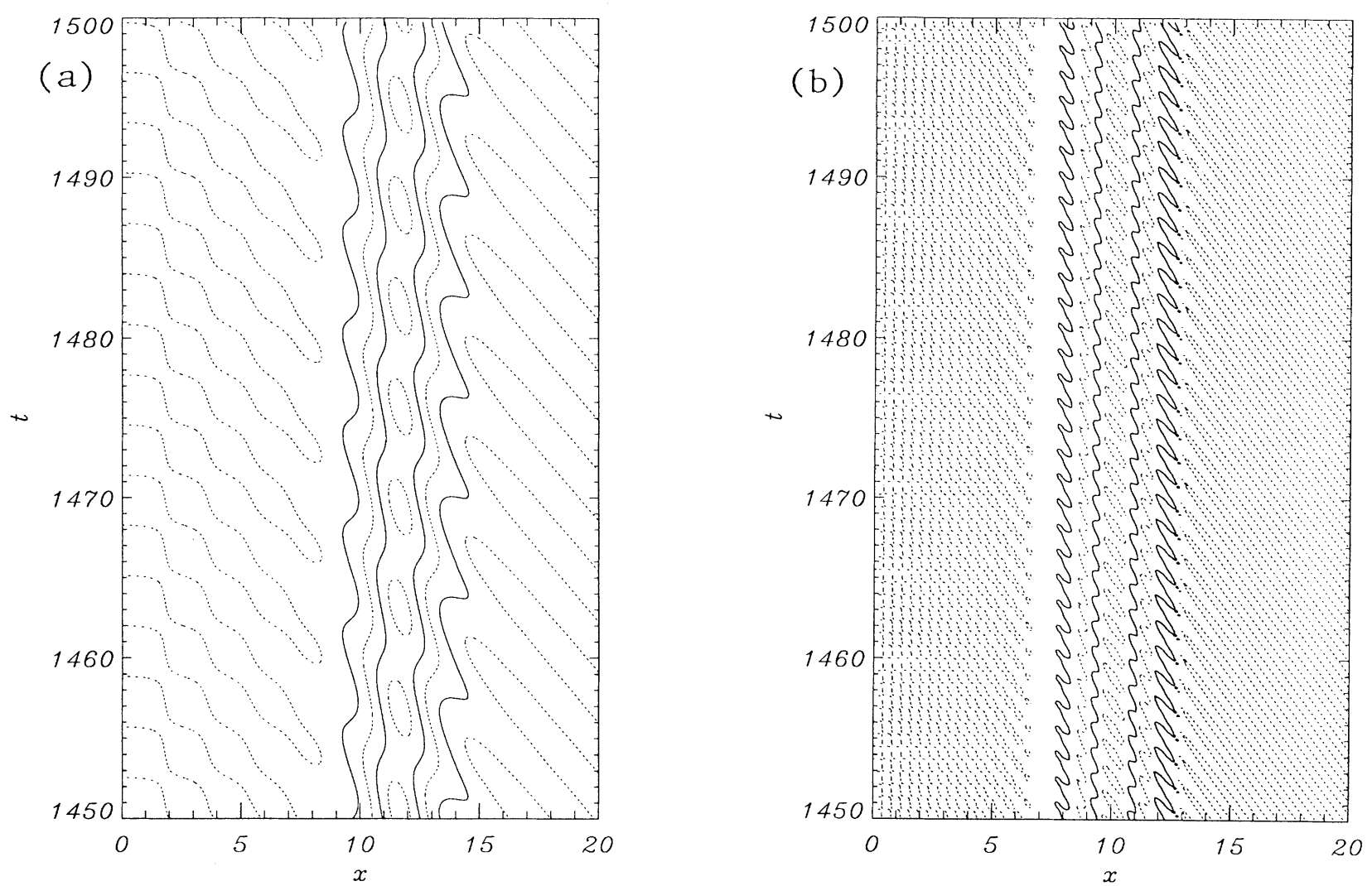

FIG. 2. Contour plots showing the details of $2 \pi$ fluxon dynamics in terms of the spatial derivative $\phi_{x}$. The applied amplitude is $a=0.5$ and the loss parameter $\alpha=0.2$. In (a) $\omega=2$, while in (b) $\omega=8$. The microwave signal is seen to influence the dynamics of the fluxon.

the frequency. These observations are in agreement with the analysis given in Sec. II. Further application of the perturbation approach described in the former section gives a good quantitative agreement.

In the following, results from numerical solution of Eq. (22) are compared to those obtained for the original SG system, Eqs. (1) and (2). From Fig. 2 the interaction between the microwave-induced radiation and the fluxon is seen to force the fluxon back and forth. The position of the fluxon has been defined as the position of the maximum value of $\phi_{x}$. Now, in order to solve Eq. (22) we have used the fluxon solution

$$
\Phi=4 \tan ^{-1} \exp \left(\frac{x-X_{0}}{\sqrt{1-\dot{X}_{0}^{2}}}\right)
$$

and the corresponding expression for the momentum,

$$
P=8 \dot{X}_{0} / \sqrt{1-\dot{X}_{0}^{2}}
$$

The potential $A(x)$ has been calculated in accordance with Eq. (15). In Fig. 3 we show a comparison between the position $X_{0}$ determined in this way (solid curve, rapidly oscillating), a resulting smoothed curve (solid curve), and the results obtained from numerical simulation of Eq. (22), i.e., the perturbation approach (dot- dashed curve). The parameter values are as in Fig. 2(b). The agreement between the smoothed and dot-dashed curves is good.

The comparison between the perturbation approach and the full numerical simulation is shown in Figs. 4 and 5 . The solid curves are the smoothed curves obtained

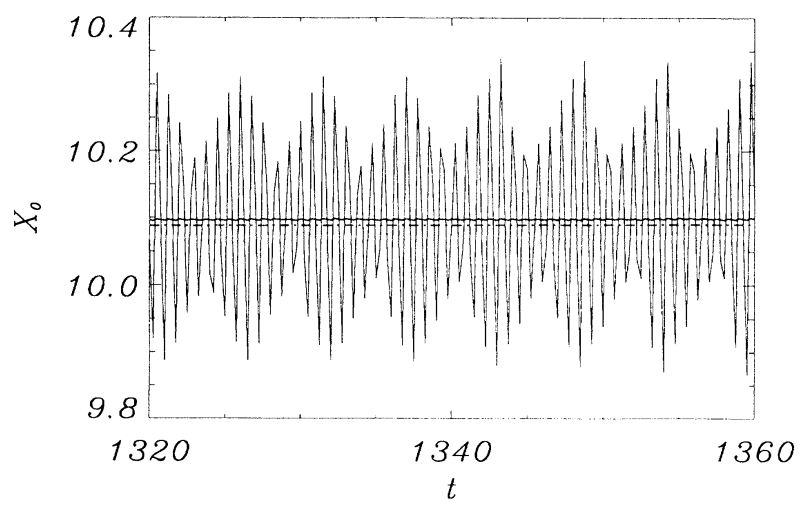

FIG. 3. Comparison between position $X_{0}$ of the $2 \pi$ fluxon obtained from full simulation of the SG equation, the rapidly oscillating function, and the value obtained from the perturbation approach, the dot-dashed curve. The solid curve is the smoothed version of $X_{0}$. The agreement between the smoothed curve and the curve obtained from the perturbation approach is good. The parameter values are $\omega=8, a=0.5$, and $\alpha=0.2$. 


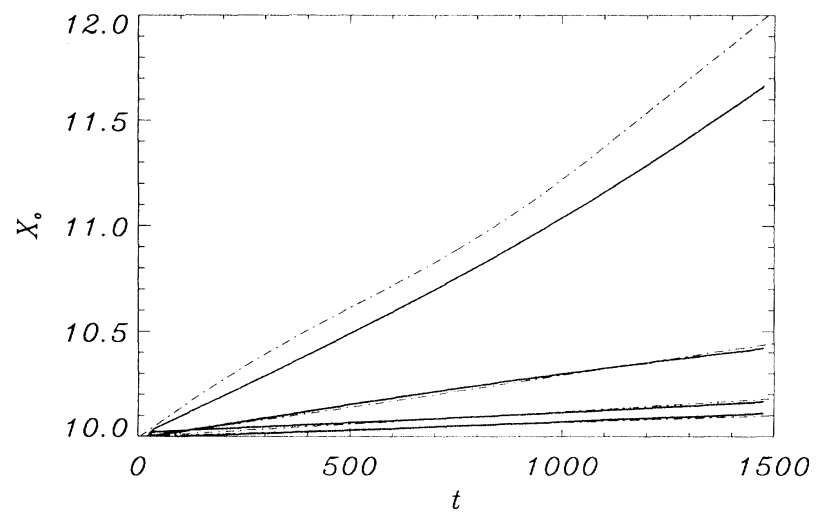

FIG. 4. Comparison between the trajectories obtained from full numerical simulation of the SG equation (solid lines) and the perturbation approach (dot-dashed lines). The parameter values are $a=0.5, \alpha=0.2$, and from the top $\omega=2,4,6,8$. For $\omega>4$ the agreement is rather good.

from the full SG system defined by Eqs. (1) and (2). The dot-dashed curves are obtained from numerical solution of Eq. (22). The parameter values in Fig. 4 are $a=0.5$ and, starting from the top curve, $\omega=2,4,6,8$. In Fig. 5 the amplitude of the applied microwave signal has been increased to $a=1$ and the frequency is $\omega=2,3,4,6,8$. The influence of the amplitude $a$ is clearly seen, in agreement with Eq. (15); i.e., the potential is proportional to $-a^{2}$. Even for smaller values of $\omega$ the qualitative agreement between the results obtained from the perturbation approach and the SG system is good. For $\omega>4$ the agreement is excellent. Finally, we note that fluxon is attracted by the active boundary and eventually absorbed as seen in Fig. 5 for $\omega=2$.

For values of the applied frequency less than unity, the averaging method described above fails. As has been shown in Ref. 3, for larger amplitudes of the driving force the low-frequency oscillation becomes localized at the active boundary, and in the nonlinear case it may be treated as an effective breather mode with the characteristic size determined by its amplitude, $\sim\left(1-\omega^{2}\right)^{-1 / 2}$. This means that a kink situated far away from the boundary does not

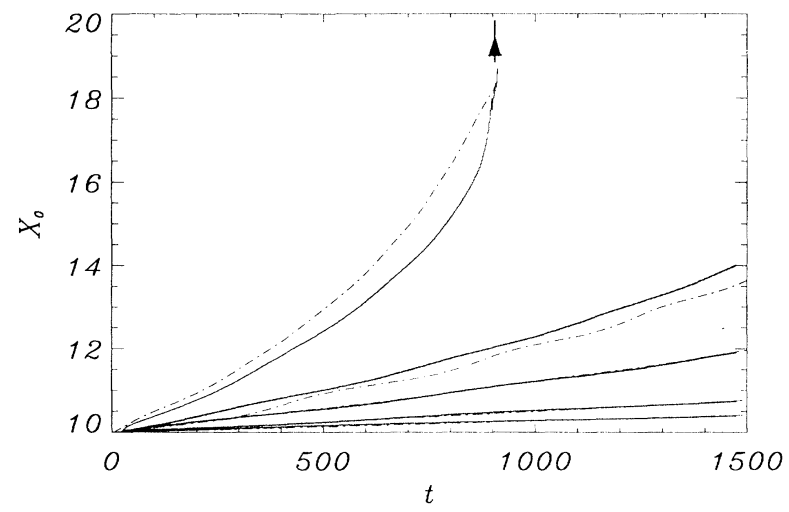

FIG. 5. Same as in Fig. 4 but for $a=1.0$, and from the top $\omega=1.5,2,4,6,8$. Again the agreement is good for $\omega>4$.

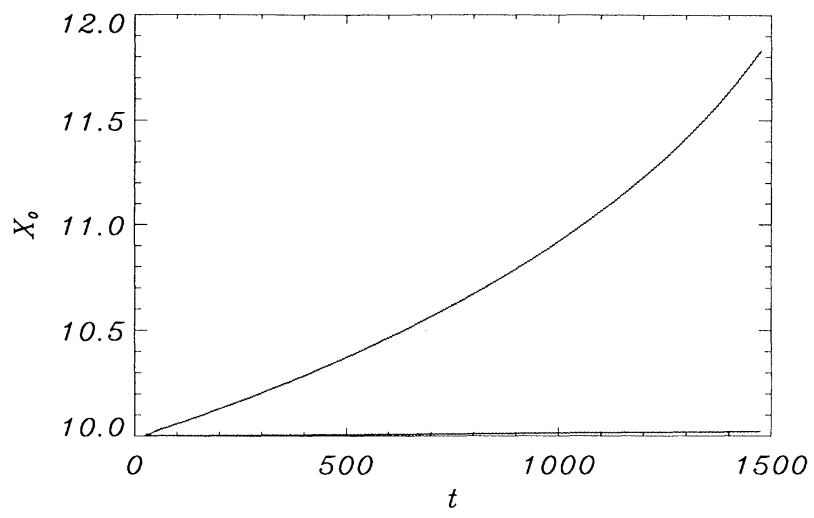

FIG. 6. Same as in Fig. 4 but for $\omega<1$, i.e., when the external frequency lies in the linear spectrum gap. The applied amplitude $a=1.0$, and from the top $\omega=0.95,0.75,0.7$. As is clearly seen, for $\omega \leq 0.7$ there is no attraction of the kink by the boundary and the kink practically does not move.

"feel" such an oscillation produced by the driving force in the junction. Figure 6 shows results of our numerical simulations for the case $\omega<1$. As may be concluded from this figure, for $\omega \leq 0.7$ the kink initially situated at $x=10$ practically does not interact with the boundary.

\section{CONCLUSION}

In conclusion, we have analyzed the influence of an $\mathrm{rf}$ signal applied to the boundary of a long Josephson junction on the dynamics of the fluxon. The model we have used is based on the SG equation including a dissipative term, with the external ac drive being taken as a boundary condition. When the frequency of the driving force is above the plasma frequency, the ac force generates a standing wave in the junction which strongly influences the fluxon motion. We have derived an averaged equation of motion to analyze the problem, and have shown that the radiation from the boundary leads to an effective oscillating potential which influences the fluxon dynamics. The effective potential decreases in the direction of the boundary where the ac force is applied, so that the fluxon always moves to the active boundary even if initially at rest. We have made a series of numerical simulations to elucidate the influence of the active boundary on the fluxon. Further we have shown that the analytical predictions and numerical simulation results are in good agreement. Thus, the physical picture based on an averaged potential for the fluxon may be indeed rather useful for understanding, even quantitatively, the physical phenomena in long Josephson junctions driven by a microwave field.

\section{ACKNOWLEDGMENTS}

Y.K. thanks Physics Laboratory I, The Technical University of Denmark, for warm hospitality during his stay there when this work was initiated. Y.K. and A.U. also acknowledge financial support by the Alexander von Humboldt-Stiftung. 
* On leave from Institute for Low Temperature Physics and Engineering, 310164 Kharkov, Ukraine. Permanent address: Optical Sciences Center, The Australian National University, ACT 2601 Canberra, Australia.

${ }^{\dagger}$ On leave from Institute of Solid State Physics, 142432 Chernogolovka, Moscow District, Russia. Present address: Il Universitá di Roma, Departimento di Fisica, 00173 Roma, Italy.

${ }^{1}$ A. Barone and G. Paterno, Physics and Applications of the Josephson Effect (Wiley, New York, 1982).

${ }^{2}$ N.F. Pedersen, IEEE Trans. Magn. 27, 3328 (1991).

${ }^{3}$ O.H. Olsen and M.R. Samuelsen, Phys. Rev. B 34, 3510 (1986).

${ }^{4}$ O.H. Olsen and M.R. Samuelsen, Phys. Lett. A 119, 391 (1987).

${ }^{5}$ O.H. Olsen and M.R. Samuelsen, Phys. Rev. B 43, 10273 (1991)
${ }^{6}$ B.A. Malomed, Phys. Lett. A 123, 494 (1987).

${ }^{7}$ Yu.S. Kivshar, O.H. Olsen, and M.R. Samuelsen, Phys. Lett. A 168, 391 (1992).

${ }^{8}$ A.V. Ustinov, J. Mygind, N.F. Pedersen, and V.A. Oboznov, Phys. Rev. B 46, 578 (1992).

${ }^{9}$ A.V. Ustinov, J. Mygind, and V.A. Oboznov, J. Appl. Phys. 72, 1203 (1992).

${ }^{10}$ Yu.S. Kivshar, A. Sánchez, and L. Vázquez, Phys. Rev. A 45, 1207 (1992).

${ }^{11}$ Yu.S. Kivshar, N. Grønbech-Jensen, and M.R. Samuelsen, Phys. Rev. B 45, 7789 (1992).

${ }^{12}$ L.D. Landau and E.M. Lifshitz, Mechanics (Pergamon, Oxford, 1960).

${ }^{13}$ Yu.S. Kivshar and B.A. Malomed. Rev. Mod. Phys. 61, 763 (1989).

${ }^{14}$ M. Salerno, M.R. Samuelsen, P.S. Lomdahl, and O.H. Olsen, Phys. Lett. 108A, 241 (1985). 